\title{
Adolescência em diferentes contextos de desenvolvimento: risco e proteção em uma perspectiva longitudinal
}

\author{
Jana Gonçalves Zappe \\ Débora Dalbosco Dell'Aglio \\ Universidade Federal do Rio Grande do Sul, RS, Brasil
}

\section{Resumo}

Este estudo investigou longitudinalmente comportamentos de risco e fatores associados em adolescentes que vivem em diferentes contextos. Participaram do estudo 503 adolescentes entre 11 e 19 anos $(\mathrm{M}=14,91$; $\mathrm{DP}=1,52)$, de ambos os sexos, que viviam com a família, em acolhimento institucional ou em internação socioeducativa. Foi utilizado o Questionário da Juventude Brasileira, administrado em dois momentos com um intervalo de 10 a 12 meses. Os adolescentes que viviam com suas famílias apresentaram os indicadores mais favoráveis nos dois momentos, os que cumpriam medida socioeducativa apresentaram os indicadores menos favoráveis e os que estavam em acolhimento institucional apresentaram uma evolução positiva ao longo do tempo. Em conclusão, enfatiza-se a importância da convivência familiar para o desenvolvimento positivo na adolescência, e a necessidade de oferecer uma intervenção qualificada em situações de institucionalização, visando à superação das vulnerabilidades presentes nestes contextos. Palavras-chave: Adolescência; comportamento de risco; institucionalização; fatores de risco; fatores de proteção.

\section{Adolescence in different contexts of development: risk and protection in a longitudinal perspective}

\begin{abstract}
This longitudinal study investigated the engagement in risk behaviors and associated factors of adolescents living in different contexts. Participants were 503 adolescents aged 11 to 19 years $(\mathrm{M}=14.91$; $\mathrm{SD}=1.52)$, of both sexes, living with family, in institutional care or juvenile institutions. The instrument used was the Questionnaire of the Brazilian Youth, administered at two moments with an interval of 10 to 12 months. Adolescents living with their families had the most favorable indicators on both occasions, those in juvenile institutions presented the least favorable indicators and those in residential care showed a positive evolution over time. In conclusion, this study emphasizes the importance of family life for the positive development in adolescence, as well as the need to offer a qualified intervention in institutionalizing situations, in order to overcome the vulnerabilities present in these contexts.
\end{abstract}

Keywords: Adolescence; risk behaviors; institutionalization; risk factors; protective factors.

\section{Adolescencia en diferentes contextos de desarrollo: riesgo y protección en una perspectiva longitudinal}

Resumen

El presente estudio investigó longitudinalmente comportamientos de riesgo y factores asociados en adolescentes que viven en diferentes contextos. Participaron 503 adolescentes entre 11 a 19 años $(\mathrm{M}=14.91, \mathrm{SD}=1.52)$, de ambos sexos, que vivían con la familia, en institución de protección o en institución para el cumplimiento de medidas socio-educativas.Fue utilizado el Cuestionario de la Juventud Brasileña, aplicado en dos etapas con un intervalo de 10 a 12 meses. Los adolescentes que vivían con sus familias presentaron los indicadores más favorables en ambas ocasiones, los que estaban en cumplimiento de medidassocioeducativaspresentaronlos indicadores menos favorables y los de instituciones de protecciónmostraronuna evoluciónpositiva al largo del tiempo. En conclusión, se enfatizala importancia de la convivenciafamiliar para el desarrollo positivo en la adolescencia, y la necesidad de ofrecer una intervención competentey cualificada en situaciones de institucionalización, con el fin de lograr la superación de las vulnerabilidades presentes en estos contextos.

Palabras clave: Adolescencia; comportamientos de riesgo; institucionalización; factores de riesgo; factores protectores. 
A adolescência compreende uma etapa do ciclo vital caracterizada por um processo que envolve mudanças físicas, psicológicas e ambientais, as quais estão diretamente relacionadas ao contexto histórico, social e cultural em que se dá o desenvolvimento (Sifuentes, Dessen, \& Oliveira, 2007). Assim, as características dos diferentes contextos de desenvolvimento promovem variadas formas de viver a adolescência, as quais marcam as trajetórias de vida de cada indivíduo e definem vulnerabilidades e potencialidades (Schoen-Ferreira, Aznar-Farias, \& Silvares, 2010). Diante disso, as atuais tendências da pesquisa sobre o desenvolvimento na adolescência enfatizam a necessidade de adotar uma perspectiva contextualista e positiva, capaz de abordar as diferentes formas de viver a adolescência em contextos variados e de colaborar com a promoção do desenvolvimento positivo do indivíduo, buscando incentivar que os adolescentes se tornem adultos capazes e comprometidos consigo mesmos, com suas famílias, comunidades e sociedade em geral (Schoen-Ferreira et al., 2010; Senna \& Dessen, 2012).

$\mathrm{Na}$ maioria das culturas, o ambiente familiar é considerado o principal contexto de desenvolvimento infanto-juvenil, aspecto legitimado por diversas resoluções nacionais e internacionais que salientam a valorização da convivência familiar (Brasil, 1990; 2009; Organização das Nações Unidas, 1989). Embora a família tenha sofrido mudanças quanto à sua estrutura e funcionamento nos últimos tempos, ela continua sendo a principal base de segurança e bem-estar de seus membros (Pratta \& Santos, 2007). Tendo em vista a existência de múltiplas possibilidades de arranjos familiares, Dessen (2010) sugere a adoção de uma definição de família como um grupo social especial, caracterizado por relações íntimas e intergeracionais entre seus membros, o que abrange as diferentes composições familiares contemporâneas. A partir disso, a família caracteriza-se pela existência de vínculos baseados na intimidade, na afetividade e nas relações intergeracionais.

Diante do reconhecimento da importância do desenvolvimento em contexto familiar, o afastamento da família e a institucionalização são admitidos apenas como recurso excepcional e breve, seja como medida protetiva ou socioeducativa. Medidas de proteção podem ser adotadas em situações de risco e vulnerabilidade social, sendo que o acolhimento institucional é a medida protetiva mais extrema, pois promove o afastamento do ambiente familiar. Medidas socioeducativas podem ser determinadas a adolescentes que cometem atos infracionais, sendo que a internação é a medida socioeducativa mais severa, pois implica em privação de liberdade e afastamento do ambiente familiar e comunitário (Brasil, 1990). A institucionalização do adolescente reflete aspectos do contexto de desenvolvimento prévio, uma vez que a adoção de medidas protetivas e socioeducativas visa à garantia da proteção integral, cuja violação está relacionada com a institucionalização. Nos casos de acolhimento institucional, há uma relação direta com a violação da garantia de proteção integral, uma vez que essa medida é adotada quando o adolescente se encontra em situação de risco ou vulnerabilidade em decorrência de negligência e/ou maus-tratos (Assis \& Farias, 2013). Com relação à internação para cumprimento de medidas socioeducativas, há uma relação indireta, pois a prática de atos infracionais é muitas vezes antecedida por uma série de violações de direitos e situações de vulnerabilidade (Dell'Aglio, Santos, \& Borges, 2004; Oliveira \& Assis, 1999).

Compreende-se que o desenvolvimento de jovens que recebem medidas de proteção ou socioeducativas é potencialmente diferente do desenvolvimento de jovens que permanecem com suas famílias, tanto antes quanto durante a institucionalização. Diferenças que podem ser observadas antes da institucionalização se devem à maior presença de riscos e violações de direitos entre adolescentes sujeitos a medidas de proteção ou de socioeducação, enquanto que durante as medidas podem ser observadas condições e oportunidades que são oferecidas pelas instituições para superar as situações de exclusão e vulnerabilidade vivenciadas, sendo esperado que saiam da instituição mais fortalecidos e em condições de dar continuidade a um desenvolvimento saudável. Diante disso, considera-se pertinente investigar o desenvolvimento em contextos familiares e institucionais, identificando as vulnerabilidades e potencialidades presentes nestes contextos, sobretudo com relação à adolescência, período decisivo do ponto de vista do desenvolvimento da autonomia e da definição da identidade adulta. Durante a adolescência, a evolução significativa de um estado de dependência infantil para a conquista progressiva de maior autonomia pelo adolescente necessita ser acompanhada por uma substituição progressiva do controle externo exercido pelos pais, educadores e outros adultos significativos para uma negociação baseada no diálogo e na confiança mútua (Pratta \& Santos, 2007). Se acompanhar o adolescente nesta transição da infância à adolescência representa um desafio às famílias, que precisam se reorganizar visando ao estabelecimento de relações cada vez mais horizontais e baseadas em negociações mútuas para promoção da autonomia, acredita-se que este desafio possa ser mais intenso quando o desenvolvimento 
durante a adolescência se dá em um contexto institucional, o qual é frequentemente mais limitado em termos de possibilidades de exercício da autonomia.

A adoção de comportamentos de risco é um aspecto que tem se destacado em estudos sobre o desenvolvimento durante a adolescência, pois envolve a participação em atividades potencialmente capazes de comprometer a saúde física e mental (Feijó \& Oliveira, 2001). A experimentação de riscos pode significar o início da adoção de hábitos não saudáveis com consequências para o desenvolvimento futuro, pois grande parte dos comportamentos habituais não saudáveis que desencadeiam doenças crônicas e outras enfermidades tem início justamente na adolescência, tais como o uso de álcool e tabaco, alimentação inadequada e sedentarismo. Estes hábitos não saudáveis estão associados ao desenvolvimento da maioria das doenças crônicas não transmissíveis, como as cardiovasculares, diabetes e câncer, as quais lideram as causas de óbito na vida adulta, no Brasil e no mundo (Brasil, 2011). Porém, apesar destes potenciais prejuízos, é possível compreender que a experimentação de riscos também possui um caráter positivo no sentido de que contribui para o estabelecimento de relações interpessoais entre pares e para o desenvolvimento da autonomia. Além disso, experimentar e explorar novas situações pode se constituir em oportunidade de aprendizado, aspecto que faz parte do desenvolvimento positivo na adolescência (Dworkin, 2005).

A maioria dos estudos que abordam diversos tipos de comportamentos salienta que eles se interrelacionam de formas complexas e possuem muitos antecedentes comuns, e por isso é recomendado o estudo de diversos comportamentos de risco conjuntamente (Guedes \& Lopes, 2010; Monshouwer et al., 2012). No Brasil, os estudos investigam comportamentos isolados e não há um programa de vigilância que monitore o conjunto de comportamentos de risco que afetam a saúde e o desenvolvimento da população jovem (Guedes \& Lopes, 2010). Recentemente, foram realizados dois levantamentos nacionais sobre fatores de risco e proteção em adolescentes escolares brasileiros, constituindo as primeiras iniciativas para conhecimento e monitoramento destes aspectos durante a adolescência, o que fornece informações úteis para a prevenção e promoção de saúde na adolescência. Através destes levantamentos, foram obtidas informações sobre comportamentos de risco em seis áreas: experimentação e uso de substâncias, comportamento sexual de risco, hábitos alimentares não saudáveis, prática inadequada de atividades físicas, comportamentos de risco no trânsito e comportamentos violentos. O levantamento mais recente (Instituto
Brasileiro de Geografia e Estatística, 2013) contou com a participação de 109.104 adolescentes escolares que cursavam o 9 ㅇ ano, a maioria com idade entre 13 e 15 anos, e revelou que $66,6 \%$ dos escolares já experimentaram bebidas alcoólicas pelo menos uma vez na vida; $19,6 \%$ já experimentaram cigarro; 7,3\% dos adolescentes já experimentaram drogas ilícitas; $28,7 \%$ dos adolescentes pesquisados já tiveram relação sexual ao menos uma vez na vida e, destes, $75,3 \%$ disse ter usado preservativo na última vez; 7,3\% dos escolares declararam envolvimento em brigas com arma branca e $6,4 \%$ com armas de fogo; $16,1 \%$ relatou não ter usado cinto de segurança quando em veículo motorizado dirigido por outra pessoa, $27,1 \%$ declararam ter dirigido veículo motorizado, embora nenhum deles tivesse a idade mínima permitida para dirigir, e 22,9\% do total de escolares admitiram ter sido transportados em veículos dirigidos por motoristas que consumiram bebida alcoólica.

$\mathrm{O}$ engajamento em comportamentos de risco na adolescência tem sido relacionado a uma série de fatores de risco e proteção pessoais e contextuais. Entre os fatores contextuais, destacam-se aspectos relacionados à família, ao grupo de pares e à rede de apoio social (especialmente escola e religião) e, como fatores pessoais, sexo, idade e características psicológicas como autoestima e autoeficácia são aspectos relevantes (Chapman, Buckley, Sheehan, Shochet, \& Romaniuk, 2011; Rafaelli, Koller, \& Cerqueira-Santos, 2012; Wang, Hsu, Lin, Cheng, \& Lee, 2010). O estudo de Wang et al. (2010), por exemplo, demonstra associação entre fatores de risco (insatisfação acadêmica, eventos de vida estressantes, percepção de comportamentos de risco no pai, na mãe e nos pares) e comportamentos de risco na adolescência (experiência sexual, ideação suicida, uso de substâncias e comportamento violento), relação que é moderada por fatores de proteção (autoestima, autoeficácia para o cuidado com a saúde e regulação emocional).

Algumas pesquisas realizadas com grupos específicos de adolescentes privados de liberdade e em situação de rua revelaram a maior vulnerabilidade para o engajamento em comportamentos de risco entre esses grupos, como uso de substâncias e comportamento sexual de risco (Muñoz-Echeverri, Noreña-Herrera, Londoño, \& Rojas-Arbeláez, 2011; Neiva-Silva, 2008; Sena \& Colares, 2008).Nesta direção, estudos longitudinais com crianças e adolescentes brasileiros em situação de vulnerabilidade, incluindo situação de rua e institucionalização, demonstraram o efeito cumulativo de fatores de risco presentes nestes contextos, impactando negativamente o desenvolvimento (Morais, 2009; Neiva-Silva, 2008; Poletto, 2011). O 
estudo de Poletto (2011), por exemplo, demonstrou que crianças e adolescentes acolhidos institucionalmente apresentaram maior número de eventos estressores e mais alto impacto dos mesmos, além de indicadores de menos bem-estar subjetivo quando comparados com crianças e adolescentes que viviam com suas famílias. No entanto, com o tempo, as crianças e adolescentes acolhidos institucionalmente apresentaram preservação ou elevação de indicadores favoráveis ao desenvolvimento, provavelmente decorrentes dos cuidados recebidos durante a institucionalização.

Diante disso, estudos envolvendo grupos específicos de adolescentes, como os institucionalizados, devem ser encorajados, uma vez que é esperado que o desenvolvimento ocorra diferentemente em contextos institucionais. Partindo disso, este estudo aborda longitudinalmente o desenvolvimento durante a adolescência considerando três contextos distintos: família, acolhimento institucional e atendimento socioeducativo, buscando identificar comportamentos de risco, fatores de risco e proteção nos diferentes contextos.

\section{MÉTODO}

\section{Participantes}

O estudo foi realizado a partir do banco de dados de uma pesquisa maior intitulada "Adolescência em Diferentes Contextos: Família e Institucionalização", que envolveu 942 adolescentes entre 11 e 19 anos $(\mathrm{M}=15,39$; D.P $=1,65)$, de ambos os sexos $(53,6 \%$ meninas e $46,4 \%$ meninos), que viviam com a família, em instituição de proteção ou em instituição para cumprimento de medidas socioeducativas. A faixa etária utilizada neste estudo levou em consideração a definição de adolescência segundo a World Health Organization (2010), a qual define essa etapa como o período entre 10 e 19 anos de idade.

Este estudo considerou os participantes que foram acessados nos dois momentos de coletas de dados, o que corresponde a 503 adolescentes, sendo 376 que viviam com suas famílias (67\% meninas e 33\% meninos), com idades entre 12 e 18 anos $(M=14,73 ; D P=1,41) ; 52$ que estavam na FASE-RS $(88,5 \%$ meninos e $11,5 \%$ meninas), com idades entre 15 e 19 anos $(M=16,94$; $\mathrm{DP}=1,06)$ e 75 que estavam em acolhimento institucional $(60 \%$ meninas e $40 \%$ meninos $)$ com idades entre 11 e 17 anos $(\mathrm{M}=14,40 ; \mathrm{DP}=1,27)$. Com relação aos adolescentes que viviam com suas famílias, a maioria morava com a mãe $(91,8 \%)$ e $63 \%$ também morava com o pai. A escolaridade dos pais era predominantemente o ensino fundamental incompleto (mãe: 26,8\%; pai: 26,4\%) e o ensino médio completo (mãe: 26,3\%; pai: 21,8\%). A maioria dos adolescentes não soube afirmar qual a renda familiar, porém, como foram acessados em escolas públicas, supõe-se que vivam com recursos socioeconômicos limitados.

\section{Procedimentos e Considerações Éticas}

O projeto de pesquisa foi aprovado pelo Comitê de Ética em Pesquisa do Instituto de Psicologia da UFRGS (protocolo n ${ }^{\circ}$ 2009060). Após a aprovação, foi realizado contato com a Secretaria Estadual de Educação e com as instituições de atendimento socioeducativo e de acolhimento institucional, solicitando concordância para a realização da pesquisa. Os adolescentes foram convidados a participar do estudo, sendo esclarecida a voluntariedade da participação, a garantia de sigilo das informações pessoais e a possibilidade de desistência a qualquer momento. As instituições que mantêm a guarda dos adolescentes assinaram o Termo de Consentimento Livre e Esclarecido (TCLE) e nas escolas também foi solicitado o TCLE aos pais, assim como os adolescentes assinaram o Termo de Assentimento. Foi disponibilizada assistência por parte da equipe de pesquisa, nos casos em que foi observada a necessidade de apoio aos participantes durante ou imediatamente após a realização da coleta de dados. A coleta de dados foi realizada em dois momentos (T1 e T2), com um intervalo de dez a doze meses.

\section{Instrumentos}

Em T1, foi utilizado o Questionário da Juventude Brasileira (Versão Fase II - Dell'Aglio, Koller, Cerqueira-Santos, \& Colaço, 2011), composto por 77 questões objetivas, sendo algumas em formato Likert. O objetivo deste questionário é investigar comportamentos de risco, fatores de risco e proteção em adolescentes, abordando também aspectos biosociodemográficos. Para a realização da pesquisa nas instituições de atendimento socioeducativo, foi construída uma versão reduzida com 47 questões, tendo em vista a dificuldade dos adolescentes para completar o instrumento original, verificada através de um estudo piloto com 10 participantes.

Em T2, foi aplicada uma versão reduzida e modificada do Questionário da Juventude Brasileira, com questões que buscaram avaliar a presença de comportamentos de risco, fatores de risco e proteção no último ano de vida dos adolescentes. A versão aplicada nas escolas e instituições de acolhimento conteve 52 questões e a versão aplicada entre adolescentes que cumpriam medidas socioeducativas conteve 33 questões.

\section{Procedimentos de Análise dos Dados}

Para avaliar os comportamentos de risco foi criada uma versão reduzida (ICR-r) do Índice de 
Comportamentos de Risco na Adolescência (ICR), o qual permite avaliar o engajamento em quatro tipos de comportamentos de risco (uso de substâncias, comportamento sexual de risco, comportamento antissocial e comportamento suicida). Estes comportamentos foram selecionados a partir de diversos estudos sobre o tema e a pontuação foi definida a partir de indicadores encontrados na literatura. O processo de elaboração e análise das propriedades psicométricas do ICR foi detalhadamente descrito em estudo específico (Alves, Zappe, \& Dell'Aglio, 2015). A versão reduzida foi criada para permitir a comparação entre os escores dos adolescentes dos três contextos de realização desta pesquisa, uma vez que os adolescentes que estavam na FASE-RS não responderam a todos os itens do Questionário da Juventude Brasileira que compõem a versão original do ICR. Assim, o ICR-r também é composto de indicadores de comportamentos de risco em quatro áreas, no entanto o número de itens é que difere da versão completa: comportamento sexual de risco (dois itens), uso de substâncias (cinco itens), comportamento antissocial (dois itens) e comportamento suicida (três itens). Ao todo, são avaliados 12 itens, sendo que cada um pode variar numa escala de 0 a 2 pontos, com uma pontuação total que pode variar de zero a 24 pontos. Quanto maior a pontuação, maior o envolvimento em comportamentos de risco. A análise das propriedades psicométricas do ICR-r revelou uma boa consistência interna $(\alpha=0,89)$.

A distribuição dos escores no ICR-r apresentou forte assimetria, de forma que optou-se pela utilização de estatísticas não-paramétricas para analisar os dados. Considerando os dados coletados em T1 (que considera ao longo da vida até o momento da coleta em T1) e T2 (que considera apenas o último ano após T1), avaliouse a variação do engajamento em comportamentos de risco e da presença de fatores de risco e proteção ao longo do tempo através de correlações de Spearman e comparações de médias através do teste de postos sinalizados de Wilcoxon para amostras relacionadas. Foram calculadas e comparadas as diferenças entre os resultados apresentados em T1 e T2 pelos adolescentes dos três contextos investigados, visando estimar se a variação ao longo de um ano foi diferente entre os três grupos.

\section{Resultados}

Todas as correlações entre os escores em comportamentos de risco nos dois tempos foram positivas e significativas. Comparações de médias indicaram que, considerando o total de participantes, houve uma diminuição no escore médio do ICR-r ao longo de um ano, assim como também diminuíram os escores em uso de substâncias, comportamento antissocial e comportamento suicida. Apenas o escore em comportamento sexual de risco aumentou, sendo estatisticamente significativas todas essas diferenças. Este mesmo padrão de variação ao longo de um ano foi identificado considerando cada contexto separadamente, mas algumas diferenças não foram estatisticamente significativas. A única exceção ao padrão de variação encontrado foi o escore médio no ICR-r entre os adolescentes que viviam com suas famílias, o qual se manteve o mesmo ao longo de um ano. Estes resultados podem ser conferidos na Tabela 1.

Contudo, é necessário relativizar as variações observadas, uma vez que, em T1 foi avaliado o engajamento em comportamentos de risco ao longo da vida em alguns itens (especialmente comportamento suicida e comportamento antissocial), e em T2 os mesmos itens avaliaram o engajamento apenas no último ano, sendo, portanto esperada uma diminuição nos escores. Com relação a comportamento sexual de risco, os itens consideraram o último ano de vida tanto em T1 como em T2, sendo que o aumento verificado pode ser então considerado como um aumento real.

Foi observado que os percentuais mais altos de adolescentes engajados em comportamentos de risco correspondem ao uso de substâncias, apresentado por $100 \%$ dos adolescentes que estavam cumprindo medida socioeducativa, $75,5 \%$ dos adolescentes que viviam com suas famílias e $62,7 \%$ dos adolescentes em acolhimento institucional. Com relação ao comportamento sexual de risco, este foi observado em $100 \%$ dos adolescentes que estavam cumprindo medida socioeducativa, $42,7 \%$ dos adolescentes que viviam com suas famílias e $38,9 \%$ dos adolescentes em acolhimento institucional. O percentual mais elevado de comportamento antissocial foi identificado entre os adolescentes que cumpriam medida socioeducativa (76,7\%), seguido dos adolescentes em acolhimento institucional $(18,7 \%)$ e dos que viviam com suas famílias (7,8\%). Adolescentes em acolhimento institucional manifestaram mais comportamento suicida $(21,3 \%)$, seguido dos que viviam com a família $(18 \%)$ e dos que cumpriam medida socioeducativa $(12,8 \%)$.

Para explorar as diferenças nos escores em comportamentos de risco nos dois momentos de coleta de dados, foram calculadas as diferenças entre as médias no ICR-r em T2 e T1 (média em T2 - média em $\mathrm{T} 1$ ), sendo os resultados organizados em três grupos conforme o escore diminuiu, aumentou ou foi mantido. Os resultados desta análise são apresentados na Tabela 2. 
TABELA 1

Variação em Comportamentos de Risco em T1 e T2

\begin{tabular}{|c|c|c|c|c|}
\hline & $\begin{array}{c}\text { Correlação entre } \\
\text { escores em T1 e T2 }\end{array}$ & Média em T1 & Média em T2 & Teste Wilcoxon \\
\hline \multicolumn{5}{|c|}{ Adolescentes vivendo com suas Famílias } \\
\hline Escore médio no ICR-r & $0,833 * *$ & 0,39 & 0,39 & 0,303 \\
\hline Comportamento Sexual de Risco & $0,811 * *$ & 0,28 & 0,55 & $9,858 * *$ \\
\hline Uso de substâncias & $0,889 * *$ & 0,64 & 0,58 & $-5,059 * *$ \\
\hline Comportamento Antissocial & $0,341 * *$ & 0,22 & 0,10 & $-4,257 * *$ \\
\hline Comportamento Suicida & $0,441 * *$ & 0,23 & 0,18 & $-2,227^{*}$ \\
\hline \multicolumn{5}{|c|}{ Adolescentes em Acolhimento Institucional } \\
\hline Escore médio no ICR-r & $0,864 * *$ & 0,51 & 0,40 & $-3,051^{*}$ \\
\hline Comportamento Sexual de Risco & $0,928 * *$ & 0,42 & 0,47 & 1,431 \\
\hline Uso de substâncias & $0,920 * *$ & 0,71 & 0,57 & $-3,869 * *$ \\
\hline Comportamento Antissocial & $0,267 *$ & 0,34 & 0,23 & $-1,031$ \\
\hline Comportamento Suicida & $0,418 * *$ & 0,40 & 0,24 & $-1,995^{*}$ \\
\hline \multicolumn{5}{|c|}{ Adolescentes cumprindo Medidas Socioeducativas } \\
\hline Escore médio no ICR-r & $0,679 * *$ & 1,19 & 1,03 & $-3,602 * *$ \\
\hline Comportamento Sexual de Risco & $0,639 * *$ & 1,16 & 1,29 & $2,403 *$ \\
\hline Uso de substâncias & $0,879 * *$ & 1,45 & 1,39 & $-1,859$ \\
\hline Comportamento Antissocial & $0,343 *$ & 1,80 & 1,29 & $-3,514 * *$ \\
\hline Comportamento Suicida & $0,495 * *$ & 0,44 & 0,20 & $-2,493 *$ \\
\hline \multicolumn{5}{|l|}{ Total } \\
\hline Escore médio no ICR-r & $0,866^{* *}$ & 0,49 & 0,46 & $-2,534 *$ \\
\hline Comportamento Sexual de Risco & $0,840 * *$ & 0,39 & 0,62 & $10,293 * *$ \\
\hline Uso de substâncias & $0,904 * *$ & 0,73 & 0,66 & $-6,558 * *$ \\
\hline Comportamento Antissocial & $0,545^{* *}$ & 0,38 & 0,23 & $-5,172 * *$ \\
\hline Comportamento Suicida & $0,439 * *$ & 0,28 & 0,19 & $-3,686^{* *}$ \\
\hline
\end{tabular}

$* p \leq 0,05 ; * * p<0,001$.

TABELA 2

Distribuição dos Adolescentes Conforme a Variação em Comportamentos de Risco (T2 - T1) por Contexto

\begin{tabular}{|c|c|c|c|c|c|c|}
\hline \multirow[t]{2}{*}{ Contexto } & \multicolumn{2}{|c|}{$\begin{array}{l}\text { Adolescentes vivendo } \\
\text { com suas Famílias }\end{array}$} & \multicolumn{2}{|c|}{$\begin{array}{c}\text { Adolescentes em } \\
\text { Acolhimento Institucional }\end{array}$} & \multicolumn{2}{|c|}{$\begin{array}{l}\text { Adolescentes cumprindo } \\
\text { Medidas Socioeducativas }\end{array}$} \\
\hline & $f$ & $\%$ & $f$ & $\%$ & $f$ & $\%$ \\
\hline \multicolumn{7}{|l|}{ Média no ICR-r } \\
\hline Manteve o escore & 79 & 21 & 19 & 25,7 & 2 & 3,9 \\
\hline Diminuiu o escore & 135 & 35,9 & 37 & 50 & 33 & 64,7 \\
\hline Aumentou o escore & 162 & 43,1 & 18 & 24,3 & 16 & 31,4 \\
\hline \multicolumn{7}{|l|}{ Comportamento Sexual de Risco } \\
\hline Manteve o escore & 234 & 63,2 & 58 & 80,6 & 23 & 45,1 \\
\hline Diminuiu o escore & 10 & 2,7 & 4 & 5,6 & 8 & 15,7 \\
\hline Aumentou o escore & 126 & 34,1 & 10 & 13,9 & 20 & 39,2 \\
\hline \multicolumn{7}{|l|}{ Uso de substâncias } \\
\hline Manteve o escore & 159 & 42,4 & 35 & 47,9 & 21 & 41,2 \\
\hline Diminuiu o escore & 134 & 35,7 & 29 & 39,7 & 18 & 35,3 \\
\hline Aumentou o escore & 82 & 21,9 & 9 & 12,3 & 12 & 23,5 \\
\hline \multicolumn{7}{|l|}{ Comportamento antissocial } \\
\hline Manteve o escore & 304 & 82,6 & 50 & 67,6 & 21 & 53,8 \\
\hline Diminuiu o escore & 51 & 13,9 & 17 & 23 & 16 & 41 \\
\hline Aumentou o escore & 13 & 3,5 & 7 & 9,5 & 2 & 5,1 \\
\hline \multicolumn{7}{|l|}{ Comportamento suicida } \\
\hline Manteve o escore & 286 & 76,9 & 49 & 67,1 & 32 & 71,1 \\
\hline Diminuiu o escore & 50 & 13,4 & 17 & 23,3 & 11 & 24,4 \\
\hline Aumentou o escore & 36 & 9,7 & 7 & 9,6 & 2 & 4,4 \\
\hline
\end{tabular}


Testes qui-quadrado indicaram que estas distribuições são diferentes e estatisticamente significativas com relação à média no ICR-r $\left(\chi^{2}=25,280 ; d f=4\right.$; $p<0,001)$, comportamento sexual de risco $\left(\chi^{2}=31,906\right.$; $d f=4 ; p<0,001)$ e comportamento infracional $\left(\chi^{2}=26,137\right.$; $d f=4 ; p<0,001)$. Nestes casos, foi possível identificar os percentuais mais elevados de adolescentes que diminuíram os escores entre os adolescentes institucionalizados.

Estas variações também foram analisadas através de ANOVA Kruskal-Wallis, comparando-se os postos de médias entre os três grupos considerando a diferença entre os escores médios em T2 e T1. Os resultados são apresentados na Tabela 3 .

A comparação múltipla entre as médias dos postos da variação no ICR-r em T2 e T1 indicou diferença estatisticamente significativa entre os adolescentes que viviam com suas famílias e os dois grupos de adolescentes institucionalizados, os quais não diferiram entre si. Isso significa que o decréscimo no escore do ICR-r em T2 foi mais alto entre os adolescentes institucionalizados. Foi identificado que o grupo de adolescentes que viviam com suas famílias apresentou uma elevação no escore em comportamento sexual de risco, diferenciando-se significativamente do grupo de adolescentes que estavam em acolhimento institucional. Com relação ao comportamento antissocial, os adolescentes que estavam na FASE-RS apresentaram uma diminuição no escore que foi diferente e estatisticamente significativa quando comparada com a diminuição apresentada pelos dois outros grupos. Os três grupos diferiram entre si com relação à diminuição no escore em comportamento suicida, a qual foi mais acentuada entre os adolescentes que estavam na FASE-RS.

Variações com relação aos fatores de risco e proteção ao longo do tempo também foram avaliadas através de correlações de Spearman e comparações de médias através do teste de postos sinalizados de Wilcoxon para amostras relacionadas. Os resultados estão apresentados na Tabela 4.

Todas as correlações entre os fatores pessoais e contextuais nos dois tempos foram positivas e significativas. Considerando o total de participantes, todos os escores diminuíram ao longo de um ano, sendo significativa a diferença entre o escore em $\mathrm{T} 1$ e o escore em T2 para todos os fatores, exceto a autoestima. Não foi identificada variação significativa na autoestima dos participantes, seja considerando o total, seja considerando cada grupo específico, o que parece demonstrar a estabilidade deste aspecto. Com relação à autoeficácia, observou-se um decréscimo significativo considerando todos os participantes e especificamente o grupo de adolescentes que viviam com suas famílias. As expectativas quanto ao futuro foram mais baixas em T2, sendo que esta variação foi significativa considerando o total de participantes e os grupos de adolescentes que viviam com suas famílias e que estavam cumprindo medidas socioeducativas.

A presença de violência intra e extrafamiliar foi menor em T2 considerando todas as situações investigadas. A presença de eventos estressores também foi menor em T2, sendo esta variação significativa em todos os casos, exceto para o grupo de adolescentes que estavam cumprindo medidas socioeducativas. De modo geral, o decréscimo verificado em violência e eventos estressores seria, de certa forma, esperado, uma vez que, em T1 foi investigada a ocorrência destes eventos ao longo da vida, enquanto que, em T2, investigou-se a ocorrência no ano anterior. Diante disso, parece que os adolescentes que estavam cumprindo medidas socioeducativas formam um grupo mais especificamente marcado por um alto número de eventos estressores, aspecto que se manteve relativamente estável ao longo de um ano.

TABELA 3

Comparações de Postos de Médias da Variação em Comportamentos de Risco em T2 e T1 por Grupo

\begin{tabular}{|c|c|c|c|c|c|c|c|c|c|}
\hline \multirow[b]{2}{*}{ Variável } & \multicolumn{2}{|c|}{$\begin{array}{c}\text { Adolescentes } \\
\text { cumprindo Medidas } \\
\text { Socioeducativas }\end{array}$} & \multicolumn{2}{|c|}{$\begin{array}{c}\text { Adolescentes } \\
\text { em Acolhimento } \\
\text { Institucional }\end{array}$} & \multicolumn{2}{|c|}{$\begin{array}{l}\text { Adolescentes vivendo } \\
\text { com suas Famílias }\end{array}$} & \multirow[t]{2}{*}{$g l$} & \multirow[t]{2}{*}{$n$} & \multirow[t]{2}{*}{$\chi^{2}$} \\
\hline & $M$ & $S D$ & $M$ & $S D$ & $M$ & $S D$ & & & \\
\hline ICR-r & $-0,15$ & 0,28 & $-0,11$ & 0,29 & $-0,01$ & 0,20 & 2 & 501 & $23,431 * *$ \\
\hline Comportamento sexual de risco & 0,14 & 0,39 & 0,05 & 0,28 & 0,28 & 0,45 & 2 & 493 & $15,451 * *$ \\
\hline Uso de substâncias & $-0,07$ & 0,24 & $-0,14$ & 0,29 & $-0,06$ & 0,21 & 2 & 499 & 5,235 \\
\hline Comportamento antissocial & $-0,47$ & 0,73 & $-0,12$ & 0,74 & $-0,12$ & 0,50 & 2 & 481 & $12,371^{*}$ \\
\hline Comportamento suicida & $-0,27$ & 0,66 & $-0,16$ & 0,68 & $-0,06$ & 0,50 & 2 & 490 & $6,480^{*}$ \\
\hline
\end{tabular}

$* p<0,05 ; * * p \leq 0,001$. 
TABELA 4

Variação em Fatores de Risco e Proteção ao Longo de Um Ano

\begin{tabular}{|c|c|c|c|c|}
\hline & $\begin{array}{l}\text { Correlação entre } \\
\text { escores em } T 1 \text { e T2 }\end{array}$ & Média em T1 & Média em T2 & Teste Wilcoxon \\
\hline \multicolumn{5}{|c|}{ Adolescentes vivendo com suas Famílias } \\
\hline Autoestima & $0,580 * *$ & 4,15 & 4,09 & $-1,892$ \\
\hline Autoeficácia & $0,492 * *$ & 3,29 & 3,22 & $-2,635^{*}$ \\
\hline Expectativas quanto ao futuro & $0,543 * *$ & 4,24 & 4,18 & $-2,669 *$ \\
\hline Violência intrafamiliar & $0,376^{* *}$ & 1,19 & 1,08 & $-6,807 * *$ \\
\hline Violência extrafamiliar & $0,393 * *$ & 1,20 & 1,09 & $-7,396 * *$ \\
\hline Eventos estressores & $0,516^{* *}$ & 3,14 & 2,26 & $-8,737 * *$ \\
\hline \multicolumn{5}{|c|}{ Adolescentes em Acolhimento Institucional } \\
\hline Autoestima & $0,432 * *$ & 4,02 & 4,04 & $-0,494$ \\
\hline Autoeficácia & $0,280 *$ & 3,18 & 3,13 & $-1,212$ \\
\hline Expectativas quanto ao futuro & $0,468 * *$ & 4,18 & 4,12 & $-1,085$ \\
\hline Violência intrafamiliar & $0,278^{*}$ & 1,83 & 1,14 & $-5,331 * *$ \\
\hline Violência extrafamiliar & $0,416^{* *}$ & 1,47 & 1,19 & $-3,991 * *$ \\
\hline Eventos estressores & $0,385 * *$ & 7,34 & 3,55 & $-6,911 * *$ \\
\hline \multicolumn{5}{|c|}{ Adolescentes cumprindo Medidas Socioeducativas } \\
\hline Autoestima & $0,527 * *$ & 4,05 & 4,06 & 0,437 \\
\hline Autoeficácia & $0,609 * *$ & 3,36 & 3,28 & $-0,374$ \\
\hline Expectativas quanto ao futuro & $0,677 * *$ & 3,96 & 3,73 & $-2,033^{*}$ \\
\hline Violência intrafamiliar & $0,326^{*}$ & 1,50 & 1,08 & $-4,474 * *$ \\
\hline Violência extrafamiliar & $0,365^{*}$ & 1,58 & 1,25 & $-3,302 * *$ \\
\hline Eventos estressores & $0,397 * *$ & 9,22 & 8,84 & $-1,271$ \\
\hline \multicolumn{5}{|l|}{ Total } \\
\hline Autoestima & $0,557 * *$ & 4,12 & 4,08 & $-1,785$ \\
\hline Autoeficácia & $0,469^{* *}$ & 3,28 & 3,21 & $-2,898^{*}$ \\
\hline Expectativas quanto ao futuro & $0,549 * *$ & 4,21 & 4,13 & $-3,418 * *$ \\
\hline Violência intrafamiliar & $0,346^{* *}$ & 1,31 & 1,09 & $-9,842 * *$ \\
\hline Violência extrafamiliar & $0,412 * *$ & 1,28 & 1,12 & $-8,837 * *$ \\
\hline Eventos estressores & $0,627 * *$ & 4,37 & 3,10 & $-10,997 * *$ \\
\hline
\end{tabular}

$* p \leq 0,05 ; * * p<0,001$.

\section{Discussão e Conclusão}

Através deste estudo, buscou-se investigar comportamentos de risco, fatores de risco e de proteção ao longo da vida e no último ano em adolescentes que vivem em diferentes contextos: família, acolhimento institucional e instituições para cumprimento de medidas socioeducativas. Os resultados apresentados indicam que existem diferenças consideráveis entre os três grupos de adolescentes, especialmente entre os jovens institucionalizados e os que viviam com suas famílias.

De modo geral, identificou-se que o engajamento em comportamentos de risco é mais alto entre os adolescentes institucionalizados que entre os adolescentes que viviam com suas famílias. Os adolescentes que estavam cumprindo medidas socioeducativas apresentaram escores mais altos com relação a comportamento antissocial, uso de substâncias e comportamento sexual de risco, enquanto os adolescentes em acolhimento institucional apresentaram escores mais altos de comportamento suicida. No entanto, observou-se que o percentual de adolescentes engajados em algum tipo de comportamento de risco é alto nos três contextos, aspecto que merece atenção e intervenções direcionadas, tendo em vista as consequências à saúde que podem advir da adoção desses comportamentos.

Resultados de análises de dados longitudinais demonstraram uma tendência geral à manutenção do engajamento em comportamentos de risco após um ano, à exceção do comportamento sexual de risco que aumentou. Este aumento provavelmente está relacionado ao desenvolvimento progressivo da sexualidade durante a adolescência, de forma que 
as experiências sexuais se tornam mais frequentes acarretando mais oportunidades para adoção de condutas de risco. No entanto, análises sobre a variação em cada um dos contextos investigados permitiram verificar um percentual mais alto de adolescentes institucionalizados que apresentaram uma diminuição dos escores ao longo do tempo. Assim, apesar de serem encontrados os percentuais mais altos de adolescentes que manifestam comportamentos de risco entre os que estavam institucionalizados, com o passar do tempo parece que a institucionalização foi protetiva no sentido de favorecer um decréscimo mais significativo que o verificado entre adolescentes que viviam com suas famílias, embora neste contexto se encontrem os adolescentes cujos resultados são os mais favoráveis.

Com relação à variação nos fatores pessoais e contextuais investigados, foi observada uma associação significativa entre os escores apresentados nos dois momentos de coletas de dados. Entre os resultados mais significativos, observou-se a estabilidade da autoestima dos participantes, independentemente do contexto considerado. Assim, parece que a definição da autoestima está mais relacionada com outras etapas do desenvolvimento psicológico, especialmente à infância, já que não foi observada variação durante a adolescência, aspecto também evidenciado em outros estudos (Hutz\& Zanon, 2011; Kernis, 2005; Sbicigo, Bandeira, \& Dell'Aglio, 2010). Também não foi observada influência do contexto na variação da autoestima, aspecto que reforça a tendência à estabilidade deste fator durante a adolescência. Uma vez que a autoestima está relacionada à satisfação de vida, à saúde mental e ao engajamento em comportamentos de risco, enfatiza-se a importância de fortalecer a autoestima em etapas precoces do desenvolvimento, sobretudo durante a infância, visando à promoção do desenvolvimento saudável em etapas posteriores, como a adolescência, dada a estabilidade deste construto.

Com relação aos demais fatores de risco e proteção, foi observado que os adolescentes que viviam com suas famílias apresentaram indicadores mais favoráveis que os adolescentes institucionalizados, os quais, por sua vez, encontram-se em situação de vulnerabilidade pela maior presença de eventos estressores e de violência, além das percepções de menos positividade nas relações com a escola e a família. Os adolescentes institucionalizados também apresentaram aspectos pessoais que desfavorecem o desenvolvimento saudável, como a autoestima e as expectativas quanto ao futuro mais baixas. No entanto, os adolescentes que estavam em acolhimento institucional apresentaram uma evolução mais positiva, pois mantiveram os escores com relação aos fatores pessoais (autoestima, autoeficácia e expectativas quanto ao futuro) e apresentaram um decréscimo nos fatores contextuais de risco (violências intra e extrafamiliar e eventos estressores). Por outro lado, os adolescentes que estavam na FASE-RS parecem ter apresentado um desenvolvimento menos favorável, uma vez que as expectativas quanto ao futuro foram mais baixas em $\mathrm{T} 2$ e se manteve o alto número de eventos estressores, apesar do decréscimo em violências intra e extrafamiliar.

Com relação aos adolescentes que viviam com suas famílias, observou-se um decréscimo na autoeficácia e nas expectativas quanto ao futuro em T2, aspectos que podem estar relacionados com a aquisição de uma perspectiva mais pessimista acerca de si e das oportunidades que o contexto oferece para atingir objetivos. Em comparação aos adolescentes de classes mais favorecidas, adolescentes de classe média e baixa são mais reticentes com relação ao futuro, pois consideram que encontram mais dificuldades para concretizar seus projetos (Liebesny \& Ozella, 2002). Esta perspectiva mais pessimista parece se intensificar ao longo da adolescência em função de que os adolescentes partem de aspirações vagas e construídas com base em normas sociais e expectativas familiares, porém, ganhando experiência, desenvolvem maior autoconhecimento e percebem com maior clareza as oportunidades oferecidas, o que leva a um refinamento de suas expectativas e aspirações com relação ao futuro (Beal \& Crockett, 2010). O estudo de Sobrosa, Santos, Oliveira e Dias (2014), sobre as expectativas em relação ao futuro profissional de adolescentes de classes socioeconômicas desfavorecidas, revelou que a maioria deles possui uma visão pessimista sobre o mercado de trabalho, visto como instável, difícil, competitivo, exigindo qualificação e desvalorizando os profissionais, de forma que o esforço pessoal é considerado o principal caminho para atingir os objetivos profissionais. Diante disso, o decréscimo identificado nas expectativas quanto ao futuro em T2 pode estar relacionado com a aquisição de uma perspectiva mais pessimista quanto às oportunidades de satisfação no trabalho, e uma percepção que confere mais valor ao esforço pessoal, o que pode ser sentido como uma pressão por resultados pessoais e se constituir em fonte de insegurança, rebaixando também a percepção de autoeficácia.

Em conjunto, os resultados do estudo evidenciam a importância de considerar as características de diferentes contextos de desenvolvimento, as quais marcam as trajetórias de vida de cada indivíduo e definem vulnerabilidades e potencialidades (SchoenFerreira et al., 2010). Pode-se concluir que a família continua ocupando um papel central no microssistema 
de desenvolvimento humano, sendo a principal base da segurança e bem-estar de seus membros (Pratta \& Santos, 2007). No entanto, é preciso reconhecer que atualmente existem diferentes tipos de composições familiares, com funcionalidades que também diferem e possuem implicações para o desenvolvimento humano (Dessen, 2010) e, nesse sentido, alguns tipos de famílias podem, ao contrário, comprometer o desenvolvimento saudável, sobretudo quando falham no exercício de suas funções ou quando são abusivas. Nestes casos, o acolhimento institucional pode representar uma alternativa para oferecer proteção e promover o desenvolvimento positivo (Arpini, 2003; Siqueira \& Dell'Aglio, 2006; Siqueira \& Dell'Aglio, 2007).

Com relação a isso, é necessário salientar que a convivência familiar deve ser sempre a primeira e mais válida alternativa, sendo as famílias fortalecidas para exercerem seus papeis de proteção e promoção do desenvolvimento dos filhos. Mesmo nos casos em que a institucionalização for inevitável, a família precisa continuar ocupando um lugar de referência, buscandose manter o contato, restaurar relações fragilizadas ou mesmo estabelecer novos laços com famílias extensas ou substitutas. Apesar de demonstrarem um desenvolvimento relativamente favorável após um ano de institucionalização, os adolescentes em acolhimento e em medidas socioeducativas ainda assim encontravam-se em desvantagem quando comparados aos adolescentes que viviam com suas famílias, o que reforça o caráter excepcional e transitório que a institucionalização deve assumir, e o investimento em prevenção e promoção do desenvolvimento saudável (Brasil, 1990; 2009).

Promover o desenvolvimento saudável durante a adolescência representa um desafio às famílias, que precisam se reorganizar visando ao estabelecimento de relações mais horizontais e baseadas em negociações mútuas para promoção da autonomia. Em um contexto institucional, acredita-se que este desafio possa ser mais intenso, pois é preciso superar as adversidades que levaram à institucionalização e estabelecer relações que possibilitem a negociação mútua e o exercício da autonomia, levando em consideração as perspectivas dos próprios adolescentes no planejamento das práticas e serviços destinados a eles.

Cabe salientar algumas limitações do estudo, as quais sugerem direções para pesquisas futuras. $\mathrm{O}$ delineamento quantitativo e a utilização de um questionário como instrumento representaram a vantagem de abordar uma ampla amostra e investigar um grande conjunto de variáveis simultaneamente. Por outro lado, essa estratégia metodológica apresenta como limitação a inviabilidade de realizar uma análise em maior profundidade, capaz de identificar sentidos e significados que as diferentes variáveis pesquisadas podem assumir para adolescentes, famílias e outras pessoas significativas. Diante desse aspecto, sugere-se a realização de estudos com delineamentos qualitativos que possam ampliar e aprofundar o conhecimento sobre comportamentos de risco e variáveis de risco e proteção, considerando as perspectivas dos participantes. Além disso, seria bastante válida a triangulação de dados, investigando o fenômeno não só a partir da perspectiva dos próprios adolescentes, mas também a partir de suas famílias e outros cuidadores significativos.

Outra limitação do estudo que precisa ser salientada decorre da comparação entre o escore cumulativo da vida e o escore do último ano em algumas variáveis, o que afeta os resultados encontrados. No entanto, resultados de análises transversais comparando os três grupos, publicadas em outro trabalho (Zappe, 2014), demonstram que os adolescentes institucionalizados diferem significativamente dos adolescentes que vivem com suas famílias quanto à maior presença de fatores de risco e ao engajamento mais alto em comportamentos de risco, além de contarem com menos fatores de proteção ao desenvolvimento, o que se verificou nos dois momentos de coleta de dados. Para superar esta limitação em pesquisas futuras, sugere-se que estudos longitudinais possam acompanhar o desenvolvimento de crianças e adolescentes por períodos de tempo mais longos, em intervalos periódicos, buscando as mesmas medidas nos diferentes tempos, evitando assim comparações entre o efeito cumulativo em uma das etapas com o efeito mais breve de um tempo mais curto em outras etapas.

Por fim, cabe recomendar fortemente o investimento em estudos que ampliem e façam avançar o conhecimento científico sobre a adolescência, assim como destes estudos derivem intervenções de altíssima qualidade visando à promoção do desenvolvimento saudável na adolescência, através da oferta de ambientes protetivos e ricos em oportunidades. É preciso lembrar que a oferta de recursos e oportunidades para o desenvolvimento saudável na adolescência, em todos os contextos de interação e especialmente naqueles com reconhecidas vulnerabilidades, acarreta implicações positivas também para a comunidade mais ampla, dada a bidirecionalidade das relações entre indivíduos e contextos. 


\section{Referências}

Alves, C. F., Zappe, J. G., \& Dell'Aglio, D. D. (2015). Índice de Comportamentos de Risco (ICR): Construção e análise das propriedades psicométricas. Estudos de Psicologia (PUCCAMP), 32(3), 371-382. http://dx.doi.org/10.1590/0103166X2015000300003

Arpini, D. M. (2003). Repensando a perspectiva institucional e a intervenção em abrigos para crianças e adolescentes. Psicologia: Ciência e Profissão, 23(1), 70-75. http://dx.doi.org/10.1590/S1414-98932003000100010

Assis, S. G. \& Farias, L. O. P. (2013). Levantamento nacional das crianças e adolescentes em serviço de acolhimento. São Paulo: Hucitec Editora.

Beal, S. J. \& Crockett, L. J. (2010). Adolescents'occupational and educational aspirations and expectations: links to high school activities and adult educational attainment. Developmental Psychology, 46(1), 258-265. http://dx.doi. org/10.1037/a0017416

Brasil. (1990, 16 de julho). Lei no 8.069, de 13 de julho de 1990. Estatuto da Criança e do Adolescente. Diário Oficial da União, p. 13.563.

Brasil. (2009, 3 de agosto). Lei no 12.010, de 3 de agosto de 2009. Diário Oficial da União, p. 1.

Brasil. Ministério da Saúde. (2011). Plano de ações estratégicas para o enfrentamento das Doenças Crônicas Não Transmissiveis (DCNT) no Brasil 2011-2022. Brasília: Ministério da Saúde. Retrieved in November 1, 2014, from http://portal.saude.gov.br/portal/arquivos/pdf/cartilha_dent_pequena_portugues_espanhol.pdf

Chapman, R. L., Buckley, L., Sheehan, M. C., Shochet, I. M., \& Romaniuk, M. (2011).The impact of school connectedness on violent behavior, transport risk-taking behavior, and associated injuries in adolescence. Journal of School Psychology, 49(4), 399-410. http://dx.doi.org/10.1016/j.jsp.2011.04.004

Dell'Aglio, D. D., Koller, S. H., Cerqueira-Santos, E., \& Colaço, V. (2011). Revisando o Questionário da Juventude Brasileira: uma nova proposta. In D. D. Dell'Aglio \& S. H. Koller (Eds.). Adolescência e Juventude: Vulnerabilidade e Contextos de Proteção (pp. 259-270). São Paulo: Casa do Psicólogo.

Dell'Aglio, D. D., Santos, S. S., \& Borges, J. L (2004). Infração juvenil feminina: uma trajetória de abandonos. Interação em Psicologia, 8(2), 191-198.

Dessen, M. A. (2010). Estudando a família em desenvolvimento: desafios conceituais e teóricos. Psicologia: Ciência e Profissão, 30(n. esp.), 202-219. http://dx.doi.org/10.1590/S1414-98932010000500010

Dworkin, J. (2005). Risk taking as developmentally appropriate experimentation for college students. Journal of Adolescent Research, 20(2), 219-241. http://dx.doi.org/10.1177/0743558404273073

Feijó, R. B. \& Oliveira, É. A. (2001). Comportamento de risco na adolescência. Jornal de Pediatria, 77(Supl. 2), S125-S134. http://dx.doi.org/10.2223/JPED.300

Guedes, D. P. \& Lopes, C. C. (2010). Validação da versão brasileira do Youth Risk Behavior Survey 2007. Revista de Saúde Pública, 44(5), 840-850. http://dx.doi.org/10.1590/S0034-89102010000500009

Hutz, C. S. \& Zanon, C. (2011). Revisão da adaptação, validação e normatização da escala de autoestima de Rosenberg. Avaliação Psicológica, 10(1), 41-49. Instituto Brasileiro de Geografia e Estatística (IBGE). (2013). Pesquisa Nacional de Saúde do Escolar 2012. Rio de Janeiro: IBGE.

Kernis, M. H. (2005). Measuring self-esteem in context: The importance of stability of selfesteem in psychological functioning. Journal of Personality, 73(6), 1569-1605. http://dx.doi.org/10.1111/j.1467-6494.2005.00359.x

Liebesny, B. \& Ozella, S. (2002). Projeto de vida na promoção de saúde. In M. L. J. Contini, S. H. Koller,\& M. N. S. Barros (Eds.). Adolescência e psicologia: Concepções, práticas e reflexões críticas (pp. 62-67). Brasília: Conselho Federal de Psicologia.

Monshouwer, K., Harakeh, Z., Lugtig, P., Huizink, A., Creemers, H. E., Reijneveld, S. A., De Winter, A. F., Van Oort, F., Ormel, J., \& Vollebergh, W. A. (2012). Predicting transitions in low and high levels of risk behavior from early to middle adolescence: The TRAILS study. Journal of Abnormal Child Psychology, 40(6), 923-931. http://dx.doi. org/10.1007/s10802-012-9624-9

Morais, N. A. de. (2009). Trajetórias de vida de crianças e adolescentes em situação de vulnerabilidade social: entre o risco e a proteção. Tese de Doutorado. Programa de Pós-Graduação em psicologia, Universidade Federal do Rio Grande do Sul. Porto Alegre: UFRGS.

Mu-oz-Echeverri, I. F., Nore-a-Herrera, C., Londo-o, B. E., \& Rojas-Arbeláez, C. A. (2011). Morbilidad atendida y conductas de riesgo de la ni-ez y adolescencia en situación de calle de Medellín, 2008. Revista de Salud Pública, 13(2), 207-218. http://dx.doi.org/10.1590/S0124-00642011000200003

Neiva-Silva, L. (2008). Uso de drogas entre crianças e adolescentes em situação de rua: um estudo longitudinal. Tese de Doutorado. Programa de Pós-Graduação em psicologia, Universidade Federal do Rio Grande do Sul. Porto Alegre: UFRGS.

Oliveira, M. B. \& Assis, S. G. (1999). Os adolescentes infratores do Rio de Janeiro e as instituições que os "ressocializam". A perpetuação do descaso. Cadernos de Saúde Pública, 15(4), 831-844. http://dx.doi.org/10.1590/ S0102-311X1999000400017

Organização das Nações Unidas (1989). Convenção dos Direitos da Criança (adotada em 20 de novembro de 1989). Assembléia Nacional das Nações Unidas. Retrieved in January 14, 2014, from http://www.unicef.org/brazil/pt/ resources_10120.htm 
Poletto, M. (2011). Bem-estar subjetivo: um estudo longitudinal com crianças e adolescentes em situação de vulnerabilidade social. Tese de Doutorado. Programa de Pós-Graduação em psicologia, Universidade Federal do Rio Grande do Sul. Porto Alegre: UFRGS.

Pratta, E. M. M. \& Santos, M. A. dos. (2007). Família e adolescência: a influência do contexto familiar no desenvolvimento psicológico de seus membros. Psicologia em Estudo, 12(2), 247-256. http://dx.doi.org/10.1590/ S1413-73722007000200005

Rafaelli, M., Koller, S. H., \& Cerqueira-Santos, E. (2012). Protective factors moderate between risk exposure and problem behaviour among low income Brazilian adolescents. British Journal of Educational Psychology, 9, 74-92.

Sbicigo, J. B., Bandeira, D. R., \& Dell'Aglio, D. D. (2010). Escala de Auto-estima de Rosenberg (EAR): Validade fatorial e consistência interna. Psico-USF, 15(3), 395-403. http://dx.doi.org/10.1590/S1413-82712010000300012

Schoen-Ferreira, T. H., Aznar-Farias, M., \& Silvares, E. F. de M. (2010). Adolescência através dos séculos. Psicologia: Teoria e Pesquisa, 26(2), 227-234. http://dx.doi.org/10.1590/S0102-37722010000200004

Sena, C. A. \& Colares, V. (2008). Comportamentos de risco para a saúde entre adolescentes em conflito com a lei. Cadernos de Saúde Pública, 24(10), 2314-2322. http://dx.doi.org/10.1590/S0102-311X2008001000012

Senna, S. R. \& Dessen, M. A. (2012). Contribuições das teorias do desenvolvimento humano para a concepção contemporânea da adolescência. Psicologia: Teoria e Pesquisa, 28(1), 101-108. http://dx.doi.org/10.1590/S010237722012000100013

Sifuentes, T. R., Dessen, M. A., \& Oliveira, M. C. S. L. (2007). Desenvolvimento humano: desafios para a compreensão das trajetórias probabilísticas. Psicologia: Teoria e Pesquisa, 23, 379-385. http://dx.doi.org/10.1590/S010237722007000400003

Siqueira, A. C. \& Dell'Aglio, D. D. (2006). O impacto da institucionalização na infância e na adolescência: Uma revisão de literatura. Psicologia \& Sociedade, 18(1), 71-80. http://dx.doi.org/10.1590/S0102-71822006000100010

Siqueira, A. C. \& Dell'Aglio, D. D. (2007). Retornando para a família de origem: Fatores de risco e proteção no processo de reinserção de uma adolescente institucionalizada. Revista Brasileira de Crescimento e Desenvolvimento Humano, 17(3), 134-146.

Sobrosa, G. M. R., Santos, A. S. dos, Oliveira, C. T. de, \& Dias, A. C. G. (2014). Perspectivas de futuro profissional para jovens provenientes de classes socioeconômicas desfavorecidas. Temas em Psicologia, 22(1), 223-234. http://dx.doi. org/10.9788/TP2014.1-17

Wang, R. H., Hsu, H. Y., Lin, S. Y., Cheng, C. P., \& Lee, S. L. (2010). Risk behaviours among early adolescents: risk and protective factors. Journal of Advanced Nursing, 66(2), 313-323. http://dx.doi.org/10.1111/j.1365-2648.2009.05159.x

World Health Organization. (2010). Adolescent job aid: a handy desk reference tool for primary level health workers. Retrieved in November 1, 2014, from http://whqlibdoc.who.int/publications/2010/9789241599962 eng.pdf

Zappe, J. G. (2014). Comportamento de risco na adolescência: aspectos pessoais e contextuais. Tese de Doutorado. Programa de Pós-Graduação em psicologia, Universidade Federal do Rio Grande do Sul. Porto Alegre: UFRGS.

Autores:

Jana Gonçalves Zappe - Doutora, Universidade Federal do Rio Grande do Sul.

Débora Dalbosco Dell'Aglio - Doutora, Universidade Federal do Rio Grande do Sul.

\author{
Endereço para correspondência: \\ Jana Gonçalves Zappe \\ Rua Evaristo Tonin, 382 - Bairro Itararé \\ 97045-180 Santa Maria, RS, Brasil \\ <janazappe@hotmail.com>
}

Recebido em: 05.08.2015

Aceito em: 03.02.2016 